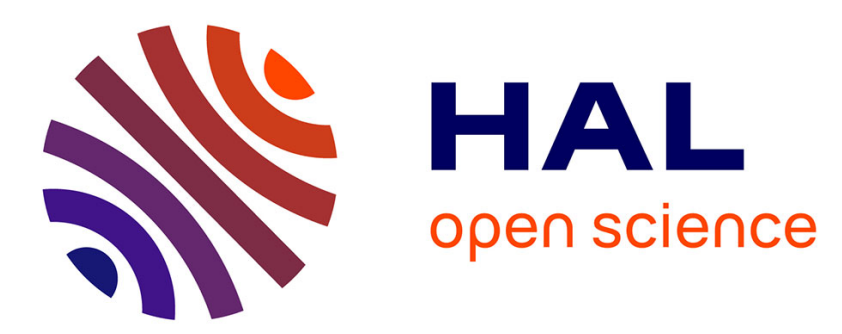

\title{
Depth Estimation for a Point Feature: Structure from motion \& Stability Analysis
}

Rayane Benyoucef, Hicham Hadj-Abdelkader, Lamri Nehaoua, Hichem Arioui

\section{To cite this version:}

Rayane Benyoucef, Hicham Hadj-Abdelkader, Lamri Nehaoua, Hichem Arioui. Depth Estimation for a Point Feature: Structure from motion \& Stability Analysis. 58th IEEE Conference on Decision and Control (CDC 2019), Dec 2019, Nice, France. pp.3991-3996, 10.1109/CDC40024.2019.9029396 . hal-02419275

\section{HAL Id: hal-02419275 \\ https://hal.science/hal-02419275}

Submitted on 19 Dec 2019

HAL is a multi-disciplinary open access archive for the deposit and dissemination of scientific research documents, whether they are published or not. The documents may come from teaching and research institutions in France or abroad, or from public or private research centers.
L'archive ouverte pluridisciplinaire $\mathbf{H A L}$, est destinée au dépôt et à la diffusion de documents scientifiques de niveau recherche, publiés ou non, émanant des établissements d'enseignement et de recherche français ou étrangers, des laboratoires publics ou privés. 


\title{
Depth Estimation for a Point Feature: Structure from motion \& Stability Analysis
}

\author{
R. Benyoucef, H. Hadj-Abdelkader, L. Nehaoua and H. Arioui
}

\begin{abstract}
This paper presents a new approach to recover the depth information from images of a monocular vision system. The depth's estimation for a point is achieved by designing a nonlinear observer based on a polytopic a structure. The fulfillment of the conditions of the state estimation, that depends on the applied velocities for the nonlinear system, is required. To this end, the observability analysis is performed to establish the kinematic conditions for the reconstruction of unmeasured states. The stability analysis is carried out using Lyapunuv theory. The observer gains were computed from the resolution of the Linear Matrix Inequality (LMI) constraints. Illustrations and simulation results are given at the end to prove the effectiveness of the proposed approach.

Keywords: Nonlinear Observers, Polytopic Systems, Feature Depth Estimation, LMI constraints, Lyapunov Theory, Structure From Motion.
\end{abstract}

\section{INTRODUCTION}

During the last decades, the observation techniques for nonlinear systems have received a large interest within control community, and many works have been devoted for the design of different observers in order to enhance the reliability and performance, since in many control applications the state vector availability is required.

A great deal of applications, where the camera is used, require in general precise knowledge of the depth. Many works have previously dealt with recovering the 3D structure for common applications, like reconstructing the 3D scene, or obtaining the pose of an object by means of different observation techniques. Filtering techniques have been used for extracting the 3D structure like an Extended Kalman Filter (EKF) [15] that is based on a local linearization of the system, which does not approximate well enough a highly nonlinear system, and cannot describe the system dynamics. Besides it requires jacobian matrices calculations, which is difficult to be derived analytically due to complicated derivatives, or numerically since it involves a high computational cost. Another filtering technique based on an Unscented Kalman Filter was presented in [16], the main drawback of this approach is being explicitly limited to gaussian probability distribution. In [10], the problem of the depth observation within classical visual servoing schemes is solved, also in [1], Structure from Motion (SfM) is addressed with active optimization of the estimation error transient response to enhance the convergence rate, by applying some constrains on the camera motion. Same thing was done in [7] using two different parametrization based on both planar

All authors are with IBISC Lab, Evry Val d'Essonne (UEVE), Paris Saclay University, 43 Rue du Pelvoux, 91080 Courcouronnes. France rayane.benyoucefduniv-evry. fr and spherical projection. However, the theoretical analysis of all these works neglect a perturbation term so that the exponential stability can be proved. In this paper, we present a novel method for depth estimation to remedy the problems stated before, considering linear parameter-varying (LPV) model of the system that defines the relation between the variation of the feature coordinates and the instantaneous camera velocity [15]. The present technique takes the inverse of the depth as an unmeasured state with known dynamics and uses Thau-Luenberger observer for T-S fuzzy systems [11] assuming a perfect knowledge of the camera intrinsic parameters. Nevertheless, in our case due to the fact that the observability condition depends on the current states and applied inputs, usually refers to non-uniformly observable system, may influence the fulfillment of the observability criteria. Thus we rely on appropriate inputs excitation so that the observation properties hold.

Designing a T-S observer is achieved when a set of criterion enabling the state reconstruction are met and this problem is usually formulated using the Linear Matrix Inequalities (LMI) [12]. Takagi-Sugeno (T-S) systems have received considerable attention in recent years [19]-[21], for the reason of its capability of representing a large class of nonlinear systems with reduced mathematical complexity. The structure can describe both discrete [24], [25] and continuous [23] systems using measurable or unmeasurable premise variables. The convergence conditions when using Takagi-Sugeno (T-S) structure is usually expressed in terms of linear matrix inequality (LMI), to obtain the observer gains. LMI formalism is used also to assign the closed loop poles in a specific region in the complex plane to guarantee a satisfactory transient convergence responses [26], [27].

This paper is organized as follows: section.II introduces basic definition and the description of the nonlinear model. Subsequently, section.III gives the T-S representation of the system and discusses the observability analysis. section.IV covers the nonlinear observer design. Experiments are conducted to discuss the performances of the proposed observer and comparing this later to the one presented in [1] in section.V, Finally section.VI draws some conclusions and future perspectives.

\section{MAthematical BACKGRound}

For better readability, we adopt the following notation: the matrices are represented in upper case bold letters $\mathbf{X}$ and vectors are in lower case bold letters $\mathbf{x}$ otherwise, the remaining notations represent scalars $(x$ or $X)$. 
Before formulating our problem, recall the following basic definitions and lemmas which will be used in the proof of our main results.

Theorem 1 (Kalman Observability Rank Condition): For every matrix $\mathbf{A} \in \mathbb{R}^{n \times n}$ and $\mathbf{C} \in \mathbb{R}^{m \times n}$. The time invariant linear system:

$$
\left\{\begin{array}{l}
\dot{\mathbf{x}}(t)=\mathbf{A x}(t) \\
\mathbf{y}(t)=\mathbf{C x}(t)
\end{array}\right.
$$

is observable if and only if the $m n \times n$ the given observability matrix $\mathbf{O}$ has a full rank of $n$. :

$$
\mathbf{O}=\left[\begin{array}{c}
\mathbf{C} \\
\mathbf{C A} \\
\vdots \\
\mathrm{CA}^{n-1}
\end{array}\right]
$$

Lemma 1: For every matrix $\mathbf{G}=\mathbf{G}^{T}>0, \mathbf{X}$ and $\mathbf{Y}$ with appropriate dimensions, the property below holds [28]:

$$
\mathbf{X}^{T} \mathbf{Y}+\mathbf{Y}^{T} \mathbf{X} \leq \mathbf{X}^{T} \mathbf{G} \mathbf{X}+\mathbf{Y}^{T} \mathbf{G}^{-1} \mathbf{Y}
$$

Lemma 2 (the Schur complement [8]): consider following convex nonlinear inequalities:

$$
\mathbf{R}>0, \quad \mathbf{Q}-\mathbf{S R}^{-1} \mathbf{S}^{T}>0
$$

where $\mathbf{Q}=\mathbf{Q}^{T}$ and $\mathbf{R}=\mathbf{R}^{T}$. Hence, the previous inequalities can be written in the following form:

$$
\left[\begin{array}{rr}
\mathbf{Q} & \mathbf{S} \\
\mathbf{S}^{T} & \mathbf{R}
\end{array}\right]>0
$$

\section{A. Conventional camera model}

Let $\mathbf{P}$ be a 3-D point of coordinates $\mathbf{P}=\left(\begin{array}{lll}X Y & Y\end{array}\right)^{\top}$ defined in the camera frame $\mathcal{F}_{c}$. Its imaging by the conventional camera is obtained through the well known pinhole model (figure 1)

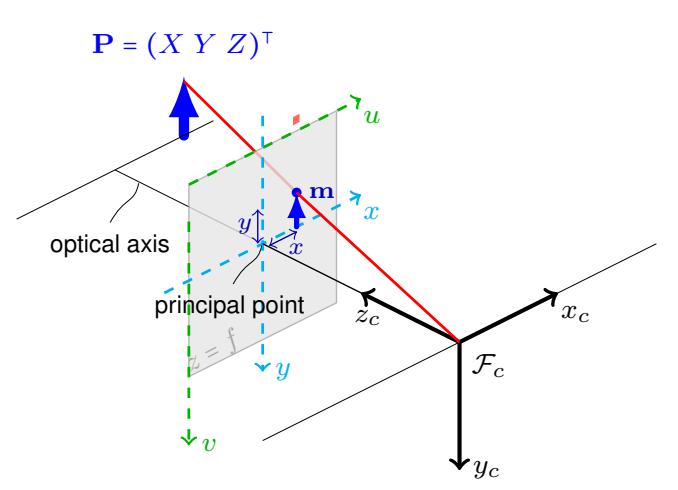

Fig. 1: Pinhole camera projection

More precisely, the 3-D point $P$ is projected in the image as a 2-D point with homogeneous coordinates given by the vector $\mathbf{m}$ as:

$$
\mathbf{m}=\left(\begin{array}{lll}
x & y & 1
\end{array}\right)^{\top}=\frac{1}{Z} \mathbf{P}
$$

The velocity of the $3 \mathrm{D}$ point $\mathbf{P}$ is related to the camera special velocity by:

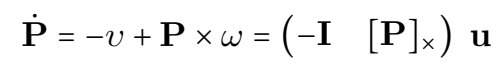

where []$_{\times}$refers to the skew-symmetric matrix of a given vector, $\mathbf{u}=\left(v^{\top} \omega^{\top}\right)^{\top}$ is the measured spatial velocity of the camera motion, with $v=\left(\begin{array}{lll}v_{x} & v_{y} & v_{z}\end{array}\right)^{\top}$ and $\omega=\left(\omega_{x} \omega_{y} \omega_{z}\right)^{\top}$ are respectively, the instantaneous linear and angular velocity of the camera frame origin. From (7), the dynamics of the inverse of the depth $\frac{1}{Z}$ is given by:

$$
\frac{d\left(\frac{1}{Z}\right)}{d t}=\left(\begin{array}{llllll}
0 & 0 & -\frac{1}{Z^{2}} & -\frac{y}{Z} & \frac{x}{Z} & 0
\end{array}\right) \mathbf{u}
$$

The time derivative of the image point $\mathbf{m}$ is linked to the camera spatial velocity $\mathbf{u}$ by the following interaction matrix [13]:

$$
\dot{\mathbf{m}}=\left(\begin{array}{cccccc}
-\frac{1}{Z} & 0 & \frac{x}{Z} & x y & -\left(1+x^{2}\right) & y \\
0 & -\frac{1}{Z} & \frac{y}{Z} & \left(1+y^{2}\right) & -x y & -x
\end{array}\right) \mathbf{u}
$$

One can note that the inverse of the depth $\frac{1}{Z}$ appears linearly in the elements of the interaction matrix corresponding to the linear velocities $v$.

Let us now define $\mathrm{X}=\left(\mathbf{s}^{\top}, \chi\right)$ as the state vector with $\mathbf{s}=\left(\begin{array}{ll}x & y\end{array}\right)^{\top} \in \mathbb{R}^{2}$ is a measurable vector, and $\chi=\frac{1}{Z} \in \mathbb{R}$ is the unmeasurable $3 \mathrm{D}$ data that we want to estimate. Using (9) and (8), the dynamics of the state vector is given by:

$$
\left\{\begin{aligned}
\dot{\mathbf{s}} & =\mathbf{f}_{m}(\mathbf{s}, \mathbf{u})+\mathbf{\Omega}^{\mathbf{T}}(\mathbf{s}, \mathbf{u}) \chi \\
\dot{\chi} & =\mathbf{f}_{u}(\mathbf{s}, \chi, \mathbf{u})
\end{aligned}\right.
$$

where the vectors $\mathbf{\Omega}^{\mathbf{T}}(\mathbf{s}, \mathbf{u}) \in \mathbb{R}^{2}, \mathbf{f}_{m}(\mathbf{s}, \mathbf{u}) \in \mathbb{R}^{2}$ and $\mathbf{f}_{u}(\mathbf{s}, \chi, \mathbf{u}) \in \mathbb{R}$ are generic and sufficiently smooth w.r.t their arguments and they are defined as:

$$
\left\{\begin{aligned}
\mathbf{f}_{m}(\mathbf{s}, \mathbf{u}) & =\left(\begin{array}{ccc}
x y & -\left(1+x^{2}\right) & y \\
1+y^{2} & -x y & -x
\end{array}\right) \omega \\
\mathbf{\Omega}^{\mathbf{T}}(\mathbf{s}, \mathbf{u}) & =\left(\begin{array}{ll}
-v_{x}+x v_{z} & -v_{y}+y v_{z}
\end{array}\right) \\
\mathbf{f}_{u}(\mathbf{s}, \chi, \mathbf{u}) & =v_{z} \chi^{2}+\left(y \omega_{x}-x \omega_{y}\right) \chi
\end{aligned}\right.
$$

In the upcoming sections, the dynamic model given in (10) will be expressed in a T-S form in order to design a polytopic Thau-Luenberger observer to estimate the depth information $\chi$.

\section{POLytopic Formulation \& OBSERVABILITy ANALYSIS}

This section presents the new formulation of the vision system model into the Takagi-Seguno (T-S) [3] and the analysis of the system uniform observabilty. 


\section{A. Sector Nonlinearities Representation}

Our aim is to design a nonlinear observer which estimates the depth information $\frac{1}{Z}$ during the camera motion. For this purpose, we adopt a new representation of the system (10), which can be expressed in the state space form given by:

$$
\left\{\begin{array}{l}
\dot{\mathbf{X}}=\mathbf{A}(\mathrm{X}, \mathbf{u}) \mathrm{X}+\mathbf{B}(\mathbf{y}) \omega \\
\mathbf{y}=\mathbf{C X}
\end{array}\right.
$$

where:

$$
\begin{gathered}
\mathbf{A}(\mathrm{x}, \mathbf{u})=\left(\begin{array}{ccc}
0 & 0 & -v_{x}+x v_{z} \\
0 & 0 & -v_{y}+y v_{z} \\
0 & 0 & \chi v_{z}+y w_{x}-x w_{y}
\end{array}\right) \\
\mathbf{B}(\mathbf{y})=\left(\begin{array}{ccc}
x y & -\left(1+x^{2}\right) & y \\
1+y^{2} & -x y & -x \\
0 & 0 & 0
\end{array}\right)
\end{gathered}
$$

and $\mathbf{y}$ represents the output of the system with:

$$
\mathbf{C}=\left(\begin{array}{lll}
1 & 0 & 0 \\
0 & 1 & 0
\end{array}\right)
$$

As said before, a T-S model is composed of a finite set of a weighted linear systems, used to achieve a trade-off between the accuracy and complexity of the model [3]-[5]. The previous system can be approximated or represented by an $r$ sub-models. The mathematical formulation of the T-S model of the system is given by:

$$
\dot{\mathrm{X}}=\sum_{i=1}^{8} \mu_{i}(\mathrm{X})\left(\mathbf{A}_{i} \mathrm{X}+\mathbf{B}(\mathbf{y}) \omega\right)
$$

where $A_{i} \in \mathbb{R}^{3 \times 3}, B \in \mathbb{R}^{3 \times 3}$ and $\mathrm{x} \in \mathbb{R}^{3 \times 1}$ is the state vector.

The weighting functions $\mu_{i}, i=1, \ldots, r$ satisfy the convex sum property expressed by:

$$
\left\{\begin{array}{c}
0 \leqslant \mu_{i} \leqslant 1 \\
\sum_{i=1}^{8} \mu_{i}=1
\end{array}\right.
$$

the system has the following three nonlinearities:

$$
h_{1}=-v_{x}+x v_{z} \quad h_{2}=-v_{y}+y v_{z} \quad h_{3}=\chi v_{z}+y w_{x}-x w_{y}
$$

These terms are bounded, so that the T-S model obtained is a weighted sum of an eight sub-models, that corresponds exactly to the nonlinear model on the considered compact bounds. For more information, please refer to [28].

\section{B. Uniform Observability Analysis}

Before going any further in the design of the observer, we should discuss the observability properties of the system. Following theorem 1 of Kalman Observability Rank Condition, the observability matrix of system (12) is expressed by:

$$
\mathbf{O}=\left[\begin{array}{ccc}
1 & 0 & 0 \\
0 & 1 & 0 \\
0 & 0 & x v_{z}-v_{x} \\
0 & 0 & y v_{z}+v_{y} \\
0 & 0 & -\left(x v_{z}-v_{x}\right)\left(v_{z} \chi-\omega_{y} x+\omega_{x} y\right) \\
0 & 0 & -\left(y v_{z}-v_{y}\right)\left(v_{z} \chi-\omega_{y} x+\omega_{x} y\right)
\end{array}\right]
$$

The system is uniformly observable if and only if $\operatorname{rank}(\mathbf{O})=3$. After a quick verification of the matrix $\mathbf{O}$, one can state that the system (12) is uniformly observable when $x v_{z}-v_{x} \neq 0$ or $y v_{z}-v_{y} \neq 0$.

This means that the linear velocities should be known and well excited to avoid the previous two conditions. In other words the feature point should be different than the optical point when the camera moves on the optical axis (figure fig:pinhole). Moreover, one can notice that the angular velocity doesn't affect the observability of the system.

\section{Design of the Polytopic Observer}

In this section we are interested in estimating the states using an adequate polytopic observer of the form:

$$
\left\{\begin{array}{l}
\dot{\hat{\mathbf{X}}}=\sum_{i=1}^{8} \mu_{i}(\hat{\mathrm{X}})\left(\mathbf{A}_{i} \hat{\mathrm{X}}+\mathbf{L}_{i}(\mathbf{y}-\hat{\mathbf{y}})\right)+\mathbf{B} \omega \\
\hat{\mathbf{y}}=\mathbf{C} \hat{\mathbf{X}}
\end{array}\right.
$$

The estimated state and output vector are respectively denoted $\hat{\mathrm{X}}$ and $\hat{\mathbf{y}}$.

Our aim then is to compute the gain $\mathbf{L}_{i}$ to ensure the convergence of the following estimation error asymptotically to zero.

$$
\mathbf{e}(t)=\mathbf{x}(t)-\hat{\mathbf{X}}(t)
$$

In what follows we omit the time dependency for simplicity sake. The dynamics of the error is modeled by:

$$
\begin{aligned}
\dot{\mathbf{e}} & =\dot{\mathrm{X}}-\dot{\hat{\mathrm{X}}} \\
& =\sum_{i=1}^{8} \mu_{i}(\mathrm{X})\left(\mathbf{A}_{i} \mathrm{X}+\mathbf{B} \omega\right)-\sum_{i=1}^{8} \mu_{i}(\hat{\mathrm{X}})\left(\mathbf{A}_{i} \hat{\mathrm{X}}+\mathbf{B} \omega+\mathbf{L}_{i}(\mathbf{y}-\hat{\mathbf{y}})\right) \\
& =\sum_{i=1}^{8} \mu_{i}(\mathrm{x}) \mathbf{A}_{i} \mathrm{X}-\sum_{i=1}^{8} \mu_{i}(\hat{\mathrm{X}})\left(\mathbf{A}_{i} \hat{\mathrm{X}}+\mathbf{L}_{i}(\mathbf{y}-\hat{\mathbf{y}})\right)
\end{aligned}
$$

then:

$$
\dot{\mathbf{e}}=\sum_{i=1}^{8} \mu_{i}(\hat{\mathrm{X}})\left(\mathbf{A}_{i}-\mathbf{L}_{i} \mathbf{C}\right) \mathbf{e}+\sum_{i=1}^{8}\left(\mu_{i}(\mathrm{X})-\mu_{i}(\hat{\mathrm{X}})\right) \mathbf{A}_{i} \mathrm{X}
$$

By replacing the expressions $\sum_{i=1}^{8} \mu_{i}(\hat{\mathrm{X}})\left(\mathbf{A}_{i}-\mathbf{L}_{i} \mathbf{C}\right)$ and $\sum_{i=1}^{8}\left(\mu_{i}(\mathrm{x})-\mu_{i}(\hat{\mathrm{X}})\right) \mathbf{A}_{i} \mathrm{X}$ by $\mathscr{A}_{\mathbf{e}}$ and $\Delta(\hat{\mathrm{X}}, \mathrm{X})$ respectively, one can obtain:

$$
\dot{\mathrm{e}}=\mathscr{A}_{\mathbf{e}} \mathbf{e}+\Delta(\hat{\mathrm{X}}, \mathrm{X})
$$

Assume that $\mathrm{X}$ and $\hat{\mathrm{X}}$ are bounded, and since $\mu_{i}(\mathrm{X}), \mu_{i}(\hat{\mathrm{X}})$ and $\mathbf{A}_{i}, i=1, \ldots, 8$ are also bounded, we can say that 
$\Delta(\hat{\mathrm{X}}, \mathrm{X})$ fulfills the Lipschitz condition in some region of interest:

$$
\Delta(\hat{\mathrm{X}}, \mathrm{X})^{T} \Delta(\hat{\mathrm{x}}, \mathrm{x})=\|\left(\Delta(\hat{\mathrm{X}}, \mathrm{x})\left\|^{2}<\gamma\right\| \hat{\mathrm{x}}-\mathrm{x}\left\|^{2}=\gamma\right\| e \|^{2}\right.
$$

the notation $\|\cdot\|$ represents the 2 -norm and $\gamma>0$ is the Lipschitz constant.

To satisfy the asymptotic stability of system (19), we draw two conditions [11]:

1) the system $\mathscr{A}_{\mathrm{e}}$ is Hurwitz.

2) $\Delta(\hat{\mathrm{X}}, \mathrm{X})$ is a vanishing disturbance i.e:

$$
\Delta(\hat{\mathrm{X}}, \mathrm{X}) \rightarrow 0 \text { when } \hat{\mathrm{X}} \rightarrow \mathrm{X}
$$

The first condition implies that there exists a positive definite symmetric matrices $\mathbf{Q} \in \mathbb{R}^{n \times n}$ and $\mathbf{P} \in \mathbb{R}^{n \times n}$ such that the following inequality holds:

$$
\mathscr{A}_{\mathbf{e}}^{T} \mathbf{P}+\mathbf{P} \mathscr{A}_{\mathbf{e}} \leqslant-\mathbf{Q}
$$

which means:

$$
\left(\mathbf{A}_{i}-\mathbf{L}_{i} \mathbf{C}\right)^{T} \mathbf{P}+\mathbf{P}\left(\mathbf{A}_{i}-\mathbf{L}_{i} \mathbf{C}\right) \leqslant-\mathbf{Q} \quad i=1, \ldots, 8
$$

Furthermore the stability analysis of the error dynamics (19) is investigated using Lyapunov theory by considering the following quadratic storage function:

$$
V=\mathbf{e}^{T} \mathbf{P e} \quad \mathbf{P}=\mathbf{P}^{T}>0
$$

Taking the time derivative of the Lyapunov function and substituting the estimation error derived previously we get:

$$
\begin{aligned}
\dot{V} & =\dot{\mathbf{e}}^{T} \mathbf{P e}+\mathbf{e}^{T} \mathbf{P} \dot{\mathbf{e}} \\
& =\left(\mathbf{e}^{T} \mathscr{A}_{\mathbf{e}}+\Delta^{T}(\hat{\mathrm{X}}, \mathrm{X})\right) \mathbf{P e}+\mathbf{e}^{T} \mathbf{P}\left(\mathscr{A}_{\mathbf{e}} \mathbf{e}+\Delta(\hat{\mathrm{X}}, \mathrm{X})\right) \\
& =\mathbf{e}^{T}\left(\mathscr{A}_{\mathbf{e}}^{T} \mathbf{P}+\mathbf{P} \mathscr{A}_{\mathbf{e}}\right) \mathbf{e}+\Delta^{T}(\hat{\mathrm{X}}, \mathrm{X}) \mathbf{P e}+\mathbf{e}^{T} \mathbf{P} \Delta(\hat{\mathrm{X}}, \mathrm{X}() 24)
\end{aligned}
$$

Recalling that the variation of the Lyapunov function must be definite negative, it follows that:

$$
\dot{V}=\mathbf{e}^{T}\left(\mathscr{A}_{\mathbf{e}}^{T} \mathbf{P}+\mathbf{P} \mathscr{A}_{\mathbf{e}}\right) \mathbf{e}+\Delta^{T}(\hat{\mathrm{X}}, \mathrm{X}) \mathbf{P e}+\mathbf{e}^{T} \mathbf{P} \Delta(\hat{\mathrm{X}}, \mathrm{X}) \leqslant 0
$$

This expression can be simplified using lemma 1, by taking $\mathbf{G}$ as a scalar denoted by $\epsilon$ :

$$
\Delta^{T}(\hat{\mathrm{X}}, \mathrm{X}) \mathbf{P e}+\mathbf{e}^{T} \mathbf{P} \Delta(\hat{\mathrm{X}}, \mathrm{X}) \leqslant \epsilon \|\left(\Delta(\hat{\mathrm{X}}, \mathrm{x}) \|^{2}+\frac{1}{\epsilon} \mathbf{e}^{T} \mathbf{P}^{2} \mathbf{e}\right.
$$

Hence, the resulting inequality is given by:

$$
\mathbf{e}^{T}\left(\mathscr{A}_{\mathbf{e}}^{T} \mathbf{P}+\mathbf{P} \mathscr{A}_{\mathbf{e}}\right) \mathbf{e}+\epsilon \Delta^{T}(\hat{\mathrm{X}}, \mathrm{X}) \Delta(\hat{\mathrm{X}}, \mathrm{X})+\frac{1}{\epsilon} \mathbf{e}^{T} \mathbf{P}^{2} \mathbf{e} \leqslant 0
$$

Since the nonlinear part defined by $\Delta(\hat{\mathrm{X}}, \mathrm{X})$ satisfies the Lipschitz condition (20), we derive the following inequality:

$$
\mathbf{e}^{T}\left(\mathscr{A}_{\mathbf{e}}^{T} \mathbf{P}+\mathbf{P} \mathscr{A}_{\mathbf{e}}+\epsilon \gamma^{2}+\frac{1}{\epsilon} \mathbf{P}^{2}\right) \mathbf{e} \leqslant 0
$$

This inequality can be expressed as LMI constrains, taking into consideration the previous equation and using the Schur complement lemma 2, one can get:

$$
\left[\begin{array}{cc}
-\mathbf{Q}+\epsilon \gamma^{2} & \mathbf{P} \\
\mathbf{P} & -\epsilon
\end{array}\right]<0
$$

The rate of convergence of the state estimation error to zero is governed by the pole placement of the matrix $\mathscr{A}_{\mathbf{e}}$. The eigenvalues can be assigned in a particular regions [17] or in a constructed region using the intersection of different LMI regions. The one chosen here defined by the combination of a disk of radius $\beta$ centered at $(0,0)$ and the half plane delimited by the value $\alpha$. i.e:

$$
S(\alpha, \beta)=\{z \in \mathbb{C}, \mathfrak{R}(z)<-\alpha,|z|<\beta\}
$$

Where $\mathfrak{R}$ denote the real part of a given complex number.

This pole clustering is governed by the following constraints:

$$
\begin{gathered}
\mathbf{P}=\mathbf{P}^{T}>0 \\
\left(\begin{array}{cc}
\beta \mathbf{P} & \mathscr{A}_{\mathbf{e}} \\
\mathscr{A}_{\mathbf{e}}^{T} \mathbf{P} & \beta \mathbf{P}
\end{array}\right)<0 \\
\mathscr{A}_{\mathbf{e}}^{T} \mathbf{P}+\mathbf{P} \mathscr{A}_{\mathbf{e}}+2 \alpha \mathbf{P}<0 \quad i=1, \ldots, 8
\end{gathered}
$$

We notice that after placing the poles we have a redundant definition of the inequalities (22) and (30c). Thus, we assume that $\mathbf{Q}=2 \alpha \mathbf{P}$.

Substituting the term $\mathscr{A}_{\mathbf{e}}$ in the previous equations, the observer gains satisfying the pole clustering are calculated under the following constraints:

$$
\begin{gathered}
\left(\begin{array}{cc}
-2 \alpha \mathbf{P}+\epsilon \gamma^{2} & \mathbf{P} \\
\mathbf{P} & -\epsilon
\end{array}\right)<0 \\
\sum_{i=1}^{8} \mu_{i}(\hat{\mathrm{X}})\left(\begin{array}{cc}
\beta \mathbf{P} & \mathbf{P}\left(\mathbf{A}_{i}-\mathbf{L}_{i} \mathbf{C}\right) \\
\left(\mathbf{A}_{i}-\mathbf{L}_{i} \mathbf{C}\right)^{T} \mathbf{P} & \beta \mathbf{P}
\end{array}\right)>0 \\
\sum_{i=1}^{8} \mu_{i}(\hat{\mathrm{x}})\left(\left(\mathbf{A}_{i}-\mathbf{L}_{i} \mathbf{C}\right)^{T} \mathbf{P}+\mathbf{P}\left(\mathbf{A}_{i}-\mathbf{L}_{i} \mathbf{C}\right)\right)+2 \alpha \mathbf{P}<0
\end{gathered}
$$

The variables must appear linearly in the constraints derived in order to obtain solvable LMIs. For this reason change of variables below is adopted: $\bar{\eta}=\epsilon \gamma^{2}$ and $\mathbf{F}_{\mathbf{i}}=\mathbf{P L}_{\mathbf{i}}$ which is equivelent to :

$$
\begin{gathered}
{\left[\begin{array}{cc}
-2 \alpha \mathbf{P}+\bar{\eta} & \mathbf{P} \\
\mathbf{P} & -\epsilon
\end{array}\right]<0} \\
\left(\begin{array}{cc}
\beta \mathbf{P} & \mathbf{P} \mathbf{A}_{i}+\mathbf{F}_{i} \mathbf{C} \\
\mathbf{A}_{i}^{T} \mathbf{P}+\mathbf{C}^{T} \mathbf{F}_{i}^{T} & \beta \mathbf{P}
\end{array}\right)>0 \\
\mathbf{A}_{i}^{T} \mathbf{P}+\mathbf{C}^{T} \mathbf{F}_{i}^{T}+\mathbf{P A}_{i}+\mathbf{F}_{i} \mathbf{C}+2 \alpha \mathbf{P} \leqslant 0 \quad i=1, \ldots, 8
\end{gathered}
$$

This implies that if there exist a a positive definite symetric matrix $\mathbf{P} \in \mathbb{R}^{3 \times 3}$, matrices $\mathbf{F}_{\mathbf{i}} \in \mathbb{R}^{3 \times 3}$, positive scalars $\bar{\eta}$ and $\epsilon$ so that the LMIs constrains to be satisfied. The resulting observer gain is given by $\mathbf{L}_{\mathbf{i}}=\mathbf{P}^{-1} \mathbf{F}_{\mathbf{i}}$.

The condition (29) guarantees the stability of the system, yet the conditions (32) allow us to place the poles in order to improve the performance of the error convergence. 


\section{SIMULATION RESULT}

In this section we report the simulation results that illustrates the depth recovering using the proposed technique, and compare this later to the one presented in [1].

Simulations are carried out using Matlab where a synthetic set of images is generated using a known camera motion. The depth information measurements $(\chi)$ of the tracked point feature as well as its image coordinates denoted by

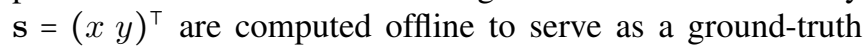
data for comparison.

The observers behavior is performed using three different initial conditions $\hat{\chi}=0.1, \hat{\chi}=0.5$ and $\hat{\chi}=1$. Two cases during the simulation are considered: with and without measurements noise.

To have a fast convergence, pole clustering taken in the region $S(\alpha, \beta)$ defined by $\beta=100$ and $\alpha=10$ shows a satisfactory response. The obtained gains calculated from the resolution of the LMIs (32) are given by ( $L_{i}$ gain matrices are given in the appendix 34$)$ :

$$
\begin{gathered}
\epsilon=10^{4} \quad \gamma=10^{-7} \\
p=10^{-10}\left(\begin{array}{ccc}
0.8951 & -0.1217 & 0.0008 \\
-0.1217 & 0.9032 & 0.0019 \\
0.0008 & 0.0019 & 0.0000
\end{array}\right)
\end{gathered}
$$

Figure (2) shows the error between the ground truth data and the T-S model of the system for $(x y \chi)$ denoted respectively by $\Delta_{x}, \Delta_{y}$ and $\Delta_{\chi}$. The order of the error indicates the perfect match between the real data and T-S model, based on which the observer is designed.

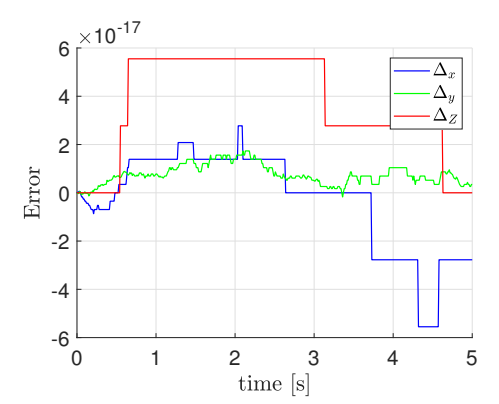

Fig. 2: Error between the ground truth data and the states of T-S model.

In the sequel we report the simulation results, for both polytopic Thau-Luenberger observer and the one proposed in [1] using a set of images that has been generated for the following applied linear/angular velocities of the camera:

$$
\begin{aligned}
& v=\left(\begin{array}{lll}
-\frac{1}{5} & \frac{1}{10} \sin \left(4 \pi t+\frac{\pi}{2}\right) & -\frac{1}{10}
\end{array}\right)^{\top} \\
& \omega=\left(\begin{array}{lll}
0 & 0 & -\frac{1}{5} \sin \left(\frac{\pi}{2} t\right)-\frac{1}{100}
\end{array}\right)^{\top} .
\end{aligned}
$$

An example of first and final images of the synthetic set are shown in figure 3 . The red dot is the tracked point, that we want to estimate its depth, and the blue line is the trajectory of the feature point when the camera is moving.

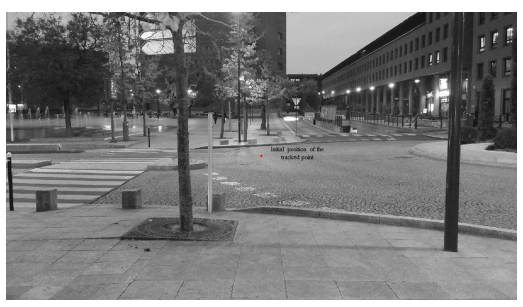

(a)

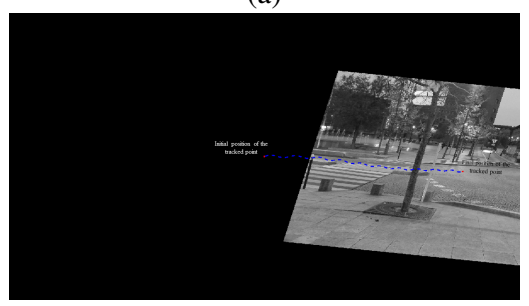

(b)

Fig. 3: Reference (a) and final synthetic image (b).

The overall comparison between our approach and the one proposed in [1] based on the results reported in the figures (4) and (5), shows that both observers converge to zero. One can note that the proposed observer converges rapidly to the real values as compared to the one presented in [1]. assigning the poles in the desired regions enabled us to get a fast convergence rate, as can be seen in the figures above, for both cases when the measurements are effected by the noise and when they are not, the observer converges to the actual values within 0.2 seconds, whereas the observer proposed in [1] attain the actual values after 2 seconds. However figures (5) illustrates that our observer is more prone to measurements noise, as for the observer proposed in [1], it shows robust response when the measurements are affected by noise.

In order to make an objective comparison between both observers, the values shown in table I, quantifies the error performance based on the Root Mean Squared Error (RMSE) formula given as:

$$
R M S E=\sqrt{\frac{1}{n} \sum_{t=1}^{n}(\chi-\hat{\chi})_{t}^{2}}
$$

TABLE I: Comparison of the Estimation Errors for both observers based on RMSE

\begin{tabular}{|c|l|l|l|}
\hline \multicolumn{2}{|c|}{ Observer } & $\begin{array}{l}\text { Result using } \\
\text { our approach }\end{array}$ & $\begin{array}{l}\text { Result using } \\
\text { the observer of } \\
{[1]}\end{array}$ \\
\hline \multirow{2}{*}{$\begin{array}{r}\text { without } \\
\text { measurements } \\
\text { noise }\end{array}$} & $\hat{\chi}=0.1$ & 0.0113 & 0.0274 \\
\cline { 2 - 4 } & $\hat{\chi}=0.5$ & 0.0257 & 0.0621 \\
\hline $\begin{array}{c}\text { with } \\
\text { noisy }\end{array}$ & $\hat{\chi}=1$ & 0.0718 & 0.1723 \\
\cline { 2 - 4 } measurements & $\hat{\chi}=0.1$ & 0.0117 & 0.0274 \\
\cline { 2 - 4 } & $\hat{\chi}=1$ & 0.0262 & 0.0621 \\
\hline
\end{tabular}

The results in tables I, indicates that the calculated RMSE values of the estimation error for the observer proposed in [1] is larger than the values computed for our observer, which 


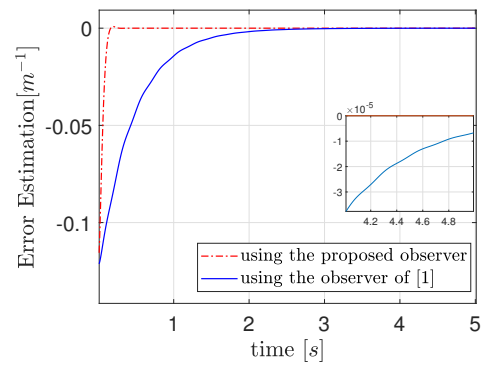

(a)

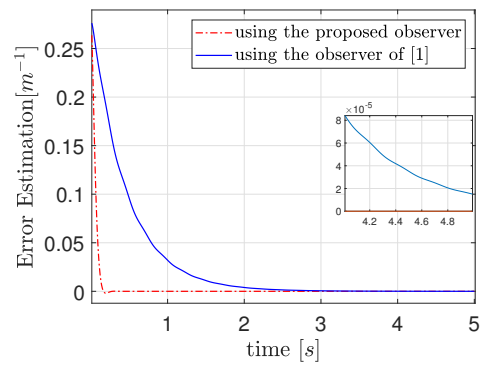

(b)

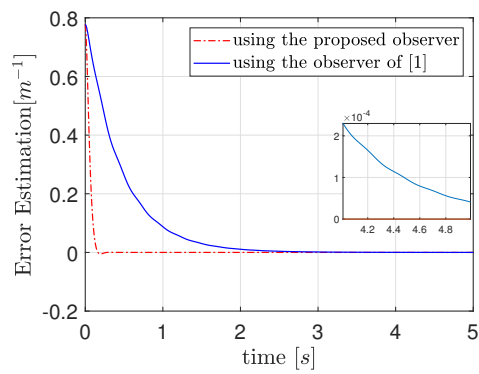

(c)

Fig. 4: Simulation results of the estimation error for the following initial values of $\hat{\chi}$ (a) $\hat{\chi}=0.1$, (a) $\hat{\chi}=0.5$ and (c) $\hat{\chi}=1$ without measurements noise.

means that, the approach presented in this paper is more accurate than the one proposed in [1].

\section{CONCLUSIONS}

In this paper, an approach consisting on designing a nonlinear observer that recovers the current value of the depth during robot/camera motion was proposed. To do a rigorous stability analysis, the nonlinear system is described by $\mathrm{T}$ $\mathrm{S}$ model with unmeasured premise variable that depend on system states. The convergence of the state estimation error is established using Lyapunov theory through a quadratic Lyapunov function candidate also to ensure a satisfactory response of the error estimation, pole clustering in a prescribed LMI region is considered along with the convergence conditions of the estimation error, and are expressed in terms of LMIs constraints then the observer gains are obtained by solving the feasibility of these LMIs. The simulations and results has proved that the proposed approach to estimate the depth of the feature point performs well comparing to

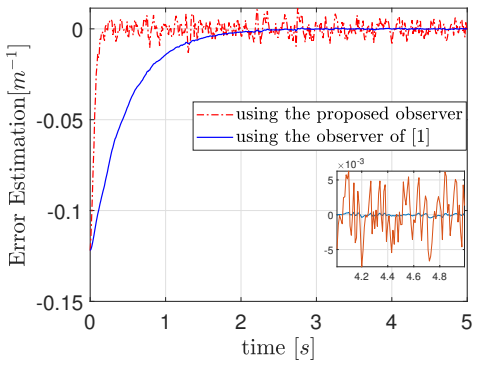

(a)

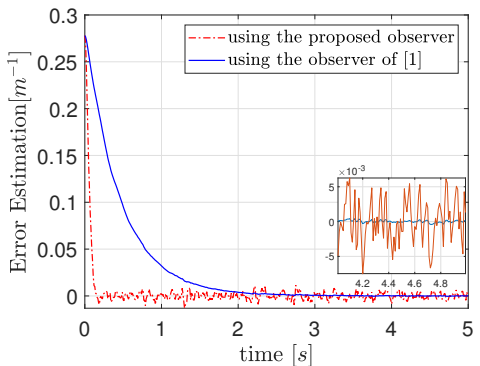

(b)

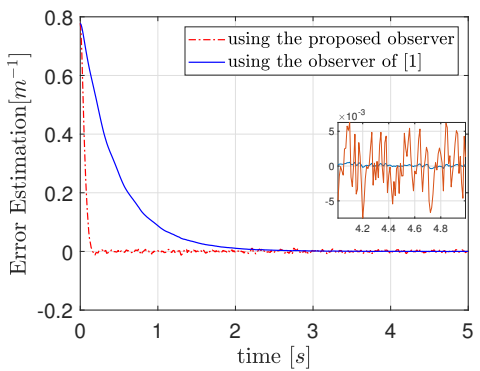

(c)

Fig. 5: Simulation results of the estimation error for the following initial values of $\hat{\chi}$ (a) $\hat{\chi}=0.1$, (b) $\hat{\chi}=0.5$ and (c) $\hat{\chi}=1$ with noisy measurements .

the approach presented in [1]. The contribution of this work consisted on giving a novelty description of the relation between the feature extracted from the image and = the linear/ angular velocity of the camera using T-S representation and synthesis a nonlinear observer without assuming approximation.

Concerning our future work we are going to consider camera auto-calibration that is to say recovering the focal length and the depth using the same observation approach proposed in the present paper also to consider more scenarios and real experiments. 


\section{APPENDIX}

$L_{1}=10^{4}\left(\begin{array}{cc}0.0089 & 0.0021 \\ 0.0037 & 0.0108 \\ -1.8819 & -1.8409\end{array}\right) L_{2}=10^{4}\left(\begin{array}{cc}0.0105 & -0.0031 \\ 0.0080 & -0.0023 \\ -3.4109 & 3.7088\end{array}\right)$
$L_{3}=10^{4}\left(\begin{array}{cc}0.0085 & 0.0021 \\ 0.0031 & 0.0106 \\ -1.8531 & -1.8538\end{array}\right) L_{4}=10^{4}\left(\begin{array}{cc}0.0103 & -0.0031 \\ 0.0075 & -0.0016 \\ -3.1634 & 3.4488\end{array}\right)$
$L_{5}=10^{4}\left(\begin{array}{cc}0.0019 & 0.0042 \\ -0.0066 & 0.0138 \\ 3.5075 & -3.2689\end{array}\right) L_{6}=10^{4}\left(\begin{array}{cc}0.0028 & -0.0046 \\ -0.0071 & -0.0007 \\ 2.9089 & 3.0488\end{array}\right)$
$L_{7}=10^{4}\left(\begin{array}{cc}0.0021 & 0.0041 \\ -0.0064 & 0.0135 \\ 3.3622 & -3.1276\end{array}\right) L_{8}=10^{4}\left(\begin{array}{cc}0.0035 & -0.0039 \\ -0.0059 & 0.0006 \\ 2.3795 & 2.5010\end{array}\right)$

\section{REFERENCES}

[1] Riccardo Spica, Paolo Robuffo Giordano. A Framework for Active Estimation: Application to Structure from Motion. IEEE Conference on Decision and Control, CDC'13, Dec 2013, Firenze, Italy. 2013.

[2] B. Espiau, F. Chaumette, P. Rives. A New Approach to Visual Servoing in Robotics. IEEE Transactions on Robotics and Automation, 8(3):313-326, 1992.

[3] T. Takagi and M. Sugeno. Fuzzy identification of systems and its applications to modelling and control, IEEE Trans. on Systems Man and Cybernetic, Volume 15, 1985, pp.116-132.

[4] H. O. Wang, K. Tanaka and M. F. Griffin. An approach to fuzzy control of nonlinear systems :stability \& design issues, IEEE Trans. on Fuzzy Systems, Volume.4, №1, 1996, pp.14-23.

[5] T.-M. Guerra and L. Vermeiren. LMI based relaxed nonquadratic stabilization conditions for nonlinear systems in the Takagi-Sugeno's form, Automatica, Volume 40, $\mathrm{N}^{\circ} 5,2004$, pp.823-829.

[6] Jeremy G. VanAntwerp, Richard D. Braatz : A tutorial on linear and bilinear matrix inequalities. Large Scale Systems Research Laboratory, Department of Chemical Engineering, University of Illinois, USA.

[7] Spica R, Robuffo Giordano P and Chaumette F (2014) Active structure from motion: application to point, sphere, and cylinder. IEEE Transactions on Robotics 30(6): 1499-1513.

[8] Matthies, L., Szelinski, R. and Kanade, T. (1989). Kalman filter-based algorithms for estimating depth from image sequences. International Journal of Computer Vision, 3: 209- 236.

[9] Smith, C. E. and Papanikolopoulos, N. P. (1994). Computation of shape through controlled active exploration. Proceedings of the 1994 IEEE International Conference on Robotics and Automation, pp. 2516-2521.

[10] Alessandro De Luca, Giuseppe Oriolo, Paolo Robuffo Giordano,'Feature Depth Observation for Image-based Visual Servoing: Theory and Experiments' The International Journal of Robotics Research Vol. 27, No. 10, October 2008, pp. 1093-1116

[11] P. Bergsten and R. Palm. Thau-Luenberger observers for TS fuzzy systems. in 9th IEEE International Conference on Fuzzy Systems, FUZZ IEEE, San Antonio, TX, USA, 2000.

[12] Jeremy G. VanAntwerp, Richard D. Braatz.A tutorial on linear and bilinear matrix inequalities.Journal of Process Control 10 (2000) 363385.

[13] François Chaumette, S. Hutchinson. Visual servo control, Part I: Basic approaches. IEEE Robotics and Automation Magazine, Institute of Electrical and Electronics Engineers, 2006, 13 (4), pp.82-90. <inria00350283>

[14] François Chaumette, S. Hutchinson. Visual servo control, Part II: Advanced approaches. IEEE Robotics and Automation Magazine, Institute of Electrical and Electronics Engineers, 2007, 14 (1), pp.109118. <inria-00350638>

[15] B. Guerreiro, P. Batista, C. Silvestre, and P. Oliveira, "Globally Asymptotically Stable Sensor-Based Simultaneous Localization and Mapping," IEEE Trans. on Robotics, vol. 29, no. 6, pp. 1380-1395, Dec 201
[16] S. Omari and G. Ducard, "Metric Visual-Inertial Navigation System Using Single Optical Flow Feature," in 2013 European Control Conference, 2013, pp. 1310-131

[17] Dalil Ichalal, Benoît Marx, José Ragot, Didier Maquin. Design of observers for Takagi-Sugeno systems with immeasurable premise variables : an L2 approach. 17th IFAC World Congress, Jul 2008, Seoul, Corée du Sud. Elsevier, pp.2768-2773, 2008, <10.3182/20080706-5KR-1001.00466>. <hal-00201030>

[18] Marino, R. and Tomei, P. (1995). Nonlinear Control Design: Geometric, Adaptive and Robust. London, Prentice-Hall.

[19] F. Blanchini and S. Miani. Stabilization of LPV systems: state feedback, state estimation, and duality. SIAM J. Control Optim., 42:76-97, 2003.

[20] G. Chesi, A. Garulli, A. Tesi, and A. Vicino. Homogeneous Polynomial Forms for Robustness Analysis of Uncertain Systems. Springer, 2009.

[21] C. Scherer. LPV control and full block multipliers. Automatica, 37:361- 375, 2001.

[22] D. Ichalal, B. Marx, J. Ragot, and D. Maquin, "State estimation of Takagi-Sugeno systems with unmeasurable premise variables," IET Control Theory Appl., vol. 4, no. 5, pp. 897-908, 2010.

[23] Dalil Ichalal, Hichem Arioui, Said Mammar. Observer design for two-wheeled vehicle : A TakagiSugeno approach with unmeasurable premise variables. 19th Mediterranean Conference on Control and Automation, MED'11, Jun 2011, Corfu, Greece. 2011. <hal-00593409>

[24] W Heemels, J. Daafouz, and M. G., "Observer-based Control of Discrete-Time LPV Systems with Uncertain Parameters," IEEE Transactions on Automatic Control, vol. 55, no. 9, pp. 21 30-21 35, 2010

[25] Peaucelle, D.; Ebihara, Y.: Robust stability analysis of discrete-time systems with parametric and switching uncertainties. In: Proceedings of the 19th World Congress of the International Federation of Automatic Control (IFAC), pp. 724-729 (2014)

[26] Rosinova, D. and Valach, P. (2014). SwitchedSystem Robust Control:Pole-PlacementLMI Based Approach,Control Conference(ICCC), 2014 15th International Carpathian,pp. 491-496

[27] M. Chilali, P. Gahinet, and P. Apkarian. Robust pole placement in LMI regions. IEEE Trans. on AC,44( 12):2257-2270,1999.

[28] Xie, L. Xie, S. and de Soza, C. Control of A Class of Uncertain Nonlinear Systems: An Observer-based Controller Design.13th IFAC World Congress, San Francisco, CA, USA,1996. 